\title{
Mapping of Neuropeptides in the Crustacean Stomatogastric Nervous System by Imaging Mass Spectrometry
}

\author{
Hui Ye, ${ }^{1}$ Limei Hui, ${ }^{2}$ Katherine Kellersberger, ${ }^{3}$ Lingjun $\mathrm{Li}^{1,2}$ \\ ${ }^{1}$ School of Pharmacy, University of Wisconsin-Madison, 777 Highland Avenue, Madison, WI 53705-2222 USA \\ ${ }^{2}$ Department of Chemistry, University of Wisconsin-Madison, 777 Highland Avenue, Madison, WI 53705-2222 USA \\ ${ }^{3}$ Bruker Daltonics Inc, 40 Manning Rd, Billerica, MA 01821-3915 USA
}

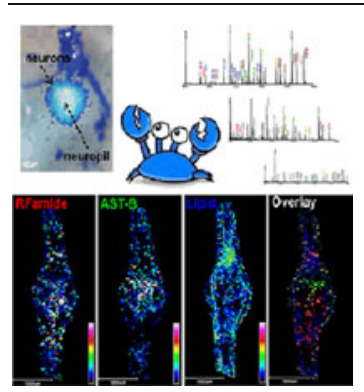

\begin{abstract}
Considerable effort has been devoted to characterizing the crustacean stomatogastric nervous system (STNS) with great emphasis on comprehensive analysis and mapping distribution of its diverse neuropeptide complement. Previously, immunohistochemistry $(\mathrm{IHC})$ has been applied to this endeavor, yet with identification accuracy and throughput compromised. Therefore, molecular imaging methods are pursued to unequivocally determine the identity and location of the neuropeptides at a high spatial resolution. In this work, we developed a novel, multifaceted mass spectrometric strategy combining profiling and imaging techniques to characterize and map neuropeptides from the blue crab Callinectes sapidus STNS at the network level. In total, 55 neuropeptides from 10 families were identified from the
\end{abstract} major ganglia in the C. sapidus STNS for the first time, including the stomatogastric ganglion (STG), the paired commissural ganglia (CoG), the esophageal ganglion (OG), and the connecting nerve stomatogastric nerve (stn) using matrix-assisted laser desorption/ionization tandem time-of-flight (MALDI-TOF/TOF) and the MS/MS capability of this technique. In addition, the locations of multiple neuropeptides were documented at a spatial resolution of $25 \mu \mathrm{m}$ in the STG and upstream nerve using MALDI-TOF/TOF and high-mass-resolution and highmass-accuracy MALDI-Fourier transform ion cyclotron resonance (FT-ICR) instrument. Furthermore, distributions of neuropeptides in the whole C. sapidus STNS were examined by imaging mass spectrometry (IMS). Different isoforms from the same family were simultaneously and unambiguously mapped, facilitating the functional exploration of neuropeptides present in the crustacean STNS and exemplifying the revolutionary role of this novel platform in neuronal network studies.

Key words: Crustacean, STNS, Neuropeptide, Imaging mass spectrometry, High resolution MS

Received: 18 May 2012/Revised: 5 September 2012/Accepted: 10 September 2012/Published online: 29 November 2012

\section{Introduction}

$\mathrm{N}^{\mathrm{e}}$ europeptides are the largest and most diverse group of endocrine signaling molecules in the nervous system. The crustacean stomatogastric nervous system (STNS) is a rich source of numerous neuropeptides and neurotransmitters [1-5]. It contains four ganglia, including a paired $\mathrm{CoG}$ and the unpaired OG and the STG [6]. The neural circuits in the STNS provide an ideal model to study how a rich repertoire of neuropeptides modulate the rhythmic motor patterns that can

Hui Ye and Limei Hui contributed equally to the maunuscript.

Electronic supplementary material The online version of this article (doi:10.1007/s13361-012-0502-z) contains supplementary material, which is available to authorized users.

Correspondence to: Lingjun Li; e-mail: 1li@pharmacy.wisc.edu control ingestive and masticatory movements of the foregut [610] to manage the different aspects of feeding, including swallowing, food storage, chewing, and the filtering of chewed food [11]. The STG, as the center of the STNS, is a ganglion of minute size and contains merely 25-30 motor neurons.

Many neuropeptides as well as neuromodulators related with rhythmic motor pattern generation in the STNS have been investigated using electrophysiological studies. For example, three families of allatostatins (AST) can reduce pyloric network burst frequency in a state-dependent manner, while application of proctolin on the STG shows robust excitatory actions [12]. However, the functions of many other neuropeptides, such as RYamide and YRamide, have not been characterized. The study of the complement and distribution of neuropeptides in this important nervous system is essential to discover the underlying mechanism of their functions [13, 14]. Previously, the CoGs and 
the STG of several crustacean species have been characterized by mass spectrometry (MS) via tissue homogenization $[15,16]$ or direct tissue analysis [17-19]. Nevertheless, spatial information of the detected neuropeptides were missing in those approaches [20], whereas a complete understanding of the functional roles of these signaling peptides in the neural circuit requires detailed characterization of all the molecular players involved in the system. A powerful technique to achieve both goals, the identification and discovery of neuropeptides and mapping the distribution of neuropeptides in the crustacean nervous system, is therefore highly demanded.

The ability to visualize neuropeptide distributions in organs or cells is usually achieved by combining immunochemistry with high-resolution microscopy [21]. However, this technique provides fairly limited chemical information and often suffers from cross-reactivity. These limitations can be overcome by imaging mass spectrometry (IMS), which integrates the high sensitivity and chemical specificity of a mass analyzer with the imaging capability of different ion sources [22]. For example, ambient ion sources such as infrared laser ablation with electrospray ionization (LAESI) [23], atmospheric pressure infrared MALDI (AP IR-MALDI) [24], desorption electrospray ionization (DESI) [25], and infrared laser ablation metastableinduced chemical ionization (IR-LAMICI) [26] have demonstrated their imaging capabilities and thereby present great potential in in vivo imaging of biomolecules. Unfortunately, their IMS applications are mostly limited by relatively low lateral-imaging resolution (typically 200-300 $\mu \mathrm{m}$ ), limited sensitivity in peptide mass range, or requirement of special instrumental modifications [22]. Alternatively, matrix-assisted laser desorption/ionization (MALDI) equipped with state-of-theart mass analyzers has been extensively employed to map a wide array of biomolecules ranging from proteins [27, 28], neuropeptides [29, 30], to drugs and metabolites [31] with excellent throughput, robustness, and sensitivity [32]. A major drawback of MALDI-IMS is its spatial resolution, which is usually limited by the laser spot diameter $(30 \sim 100 \mu \mathrm{m})$ on commercial instruments [33]. Nevertheless, advancements in sample-handling and mass spectrometers such as specially-designed confocal type objectives [34], defocused laser beam employed in "microscope" mode MALDI-MS coupled with a positionsensitive detector [35], and an imaging acquisition method "oversampling" have allowed imaging at cellular-length scales in several studies [36]. Despite the limitation of spatial resolution, MALDI-IMS has exhibited its power in neuropeptide mapping from complex neural tissues ranging from mammalian tissues such as rat spinal cord [30], brain [29, 37], pituitary gland [38] to invertebrates like the crab Cancer borealis [39, 40] and insects like the house cricket Acheta domesticus [41]. These studies exemplified the advantages of IMS in neuropeptide mapping applications: it does not require prior knowledge about the specific signaling molecules; it can distinguish between multiple isoforms in the same peptide family, and enables simultaneous mapping of numerous neuropeptides in a single experiment [33]. Mass analyzers are critical for unequivocal identification of neuropeptides from complex samples. MALDI-TOF/TOF has become a major instrument employed in MALDI-IMS applications, offering high sensitivity, wide mass range, and fast analysis speed [33]. In the current study, these advantages and the in situ MS/MS capability were demonstrated. Nevertheless, high-performance instruments like FT-ICR [18, 37, 38] or Orbitrap [41, 42] outshine by allowing for imaging multiple peptides of close masses unambiguously from tissue. The high measurement accuracy and resolving power of the FT-ICR employed in this study significantly enhanced our confidence in assigning neuropeptides based on mass-matching and resolved MS images of the neuropeptides with close masses present in tissue. Subsequent tandem MS measurements by ESI-Q-TOF further confirmed previous neuropeptide identifications obtained from the MALDI-MS platform. This novel multi-faceted mass spectrometry (MS)-based strategy combines high sensitivity, high resolving power, high mass accuracy and fast analysis speed, enabling a large-scale characterization and mapping of endogenous neuropeptides at a spatial resolution of $25 \mu \mathrm{m}$ from a small yet complex neuronal network such as the $C$. sapidus STG exemplified in this report.

For the first time, a combination of MALDI-TOF/TOF and MALDI-FT-ICR was employed to study the identity and localization of neuropeptides in the STG, an organ of minute size, with high spatial resolution and high spectral quality. The colocalization of multiple peptide isoforms from several peptide families was demonstrated in this ganglion. IMS was also performed to map the whole STNS. In addition to imaging, multiple sample preparation techniques including direct tissue analysis, tissue extract analysis, and online-ultra performance liquid chromatography (UPLC) analysis followed by tandem MS were implemented in this multi-faceted MS-based strategy. A total of 55 neuropeptides were unequivocally identified from the C. sapidus STNS in a high throughput fashion and verified by tandem MS sequencing.

\section{Experimental}

\section{Chemicals and Materials}

Methanol, acetonitrile, and formic acid (FA) were purchased from Fisher Scientific (Pittsburgh, PA, USA). 2,5-Dihydroxybenzoic acid (DHB) was obtained from MP Biomedicals, Inc. (Solon, OH, USA). Methylene blue staining solution was purchased from Sigma Aldrich (St. Louis, MO, USA). Acidified methanol was prepared using $90 \%$ methanol, $9 \%$ glacial acetic acid, and $1 \%$ water. Composition of physiological saline is $440 \mathrm{mM} \mathrm{NaCl} ; 11 \mathrm{mM} \mathrm{KCl}$; $13 \mathrm{mM} \mathrm{CaCl}_{2} ; 26 \mathrm{mM} \mathrm{MgCl}_{2} ; 10 \mathrm{mM}$ HEPES acid; $\mathrm{pH}$ 7.4-7.5. All water used in this study was Milli-Q water from a Millipore filtration system (Bedford, MA, USA).

\section{Animal Dissection}

Blue crabs C. sapidus were purchased from the local market and maintained in recirculating artificial seawater aquaria at 
ambient temperature (approximately $12-13{ }^{\circ} \mathrm{C}$ ) before use. Crabs were anesthetized by packing them on ice for $15 \mathrm{~min}$, after which the dorsal carapace were removed from each individual and its STNS were dissected free from surrounding muscle and connective tissues in chilled (approximately $10{ }^{\circ} \mathrm{C}$ ) physiological saline. The details of dissection were described elsewhere [43]. Following dissection, the STNS were immediately prepared for direct tissue analysis and IMS or saved in acidified methanol at $-80{ }^{\circ} \mathrm{C}$ for future use in peptide extraction.

\section{Direct Tissue Analysis}

For direct tissue analysis of the STNS, the STG, the stn, the $\mathrm{CoG}$, and the $\mathrm{OG}$ were removed from the STNS by microdissection scissors (Fine Science Tools, Inc., Foster City, CA, USA), briefly rinsed in acidified methanol, and desalted in $10 \mathrm{mg} / \mathrm{mL}$ aqueous 2,5-dihydroxybenzoic acid (DHB) solution as described previously [17]. The tissue ( $n=$ 3) was then placed on the MALDI plate, and $0.3 \mu \mathrm{L}$ of $150 \mathrm{mg} / \mathrm{mL}$ DHB solution (50:50 methanol:water, vol:vol) was applied on top of the tissue. Direct tissue mass spectrometric analysis was performed using a 4800 MALDI-TOF/TOF mass spectrometer (Applied Biosystems, Framingham, MA, USA).

\section{MALDI-IMS Sample Preparation}

The STNS was rinsed briefly in deionized water immediately following dissection to reduce the salt content, after which the excessive water was removed by KimWipes and the STNS was placed on a MALDI plate. For highresolution imaging experiment, the STG was removed from the STNS by microdissection scissors (Fine Science Tools, Inc., Foster City, CA, USA) and placed on an indium tin oxide (ITO)-coated glass slide. Optical images of the STNS or STG were taken by scanning the glass slide with an office scanner (Epson V300 PHOTO) in professional mode with 2400 dpi resolution. The STNS or STG was then dehydrated in a desiccator in $-20{ }^{\circ} \mathrm{C}$ for $30 \mathrm{~min}$ prior to matrix application. For matrix application, $150 \mathrm{mg} / \mathrm{mL}$ DHB (50:50 methanol:water, vol:vol) was used and the airbrush (Paasche Airbrush Company, Chicago, IL, USA) was held $35 \mathrm{~cm}$ from the plate for regular spray. Five coats were applied, and the spray duration for each coat was $30 \mathrm{~s}$ with 1 min dry time between each cycle. Alternatively for IMS of the STG $(n=3)$, the airbrush was held $45 \mathrm{~cm}$ from the plate for dryer spray. This modification allowed more matrix solvent to evaporate before reaching the plate and the formation of smaller matrix crystals on tissue surface for high resolution IMS. IMS of the STNS $(n=3)$ was performed using a 4800 MALDI TOF/TOF mass spectrometer (Applied Biosystems, Framingham, MA, USA), whereas IMS of the STG was conducted using autoflex III MALDI-TOF/TOF (Bruker Daltonics, Billerica, MA, USA) and SolariX MALDI FTICR (Bruker Daltonics, Billerica, MA, USA).

\section{MALDI-TOF/TOF MS}

A model 4800 MALDI TOF/TOF analyzer equipped with a $200 \mathrm{~Hz}, 355 \mathrm{~nm} \mathrm{Nd}: Y A G$ laser (spot diameter of $75 \mu \mathrm{m}$ ) was employed for direct tissue analysis of the STG, stn, CoG, and OG, and IMS of the STNS. Instrument parameters were set using the 4000 Series Explorer Software (Applied Biosystems, Framingham, MA, USA). All mass spectra were collected in the positive ion reflectron mode. Mass calibration was performed externally using a mixture of synthetic peptide standards. Each representative profiling mass spectrum of the STG, stn, CoG, and OG was averaged from 500 laser shots, and the mass spectra data were extracted over a mass range of $m / z 600-1800$ where the majority of the neuropeptides were detected. Tandem mass spectra (MS/MS) were achieved in situ by $2 \mathrm{kV}$ collision induced dissociation (CID) using air as the collision gas. Seven hundred fifty laser pulses were averaged for each MS/MS spectrum, and sequence interpretation was performed manually.

IMS acquisition of the STNS was controlled using the 4800 Imaging Application software (Novartis, Basel, Switzerland) available through the MALDI IMS website (www.maldimsi.org). Arrays of spectra were collected at $100 \mu \mathrm{m}$ intervals in both $\mathrm{x}$ and $\mathrm{y}$ dimensions, and each spectrum was averaged from 200 laser shots. The mass spectra data was extracted over a mass range of $m / z 600-1800$. The image files were processed and MS images of neuropeptides were generated using the TissueView software package (Applied Biosystems, Framingham, MA, USA).

IMS of the STG was performed at high lateral resolution on autoflex III MALDI-TOF/TOF mass spectrometer equipped with a $200 \mathrm{~Hz}$ smartbeam (diameter as small as $25 \mu \mathrm{m})$. The following parameters were adopted in the positive reflectron mode for imaging acquisition: ion source 1 voltage $19.00 \mathrm{kV}$, ion source 2 voltage $16.62 \mathrm{kV}$, reflector 1 voltage $20.90 \mathrm{kV}$, reflector 2 voltage $9.64 \mathrm{kV}$, and lens voltage $8.70 \mathrm{kV}$. The mass spectra data was acquired over a mass range of $m / z 600-1800$ where the majority of the neuropeptides were detected. Each spectrum consists of 500 laser shots and the array of spectra was collected at $25 \mu \mathrm{m}$ intervals in both $\mathrm{x}$ and $\mathrm{y}$ dimensions across the surface of the STG. The image file was processed and MS images of neuropeptides were generated using the flexImaging software package (Bruker Daltonics, Billerica, MA, USA).

\section{MALDI-FT-ICR MS}

MALDI-FTMS measurements were performed at high spectral resolution and high lateral resolution on a $12 \mathrm{~T}$ SolariX (Bruker Daltonics, Billerica, MA, USA) FT-ICR MS equipped with dual ESI-MALDI source and smartbeam II $1 \mathrm{kHz}, 355 \mathrm{~nm}$ solid state Nd:YAG laser focused to a diameter of $\sim 25 \mu \mathrm{m}$. Mass spectra were accumulated in positive ion mode and each spectrum consisted of 1 scan of 1000 laser shots from each spot, accumulated externally prior to measurement in the ICR cell over the mass range of 
$m / z 250-4000$. The array of spectra was collected at $25 \mu \mathrm{m}$ intervals in both $\mathrm{x}$ and $\mathrm{y}$ dimensions across the surface of the STG. Data were acquired and analyzed using the flexImaging software (Bruker Daltonics, Billerica, MA, USA), and digital images of the prepared sections were acquired prior to matrix coating.

\section{Tissue Extraction}

Fifteen STGs were pooled, homogenized, and extracted with acidified methanol as described previously [44]. Extracts were concentrated in a Savant SC 110 Speedvac concentrator (Thermo Electron Corporation, West Palm Beach, FL, USA). The extract was subsequently resuspended in $0.1 \%$ formic acid. The resuspended extracts were then vortexed and briefly centrifuged. The resulting solution was purified and concentrated with C18 ZipTip (Millipore, Billerica, MA, USA). Briefly, the ZipTip C18 was first wetted using a ACN:water:formic acid (50:49.9:0.1; vol/vol/vol) solution and then pre-equilibrated for sample binding with $0.1 \%$ formic acid in water. Subsequently, the tissue extract was loaded on the ZipTip C18. After washing with $0.1 \%$ formic acid in water for three times, the sample was eluted with 5 $\mu \mathrm{L}$ ACN:water:formic acid (50:49.9:0.1; $\mathrm{vol} / \mathrm{vol} / \mathrm{vol})$. Next, the eluent was dried and resuspended in $0.1 \%$ formic acid in water, and subjected to analysis by liquid chromatography-electrospray ionization-quadruple-time of flight (LC-ESI-Q-TOF).

\section{NanoLC-ESI-Q-TOF MS/MS}

Nanoscale LC-ESI-Q-TOF MS/MS was performed by a Waters nanoAcquity Ultra Performance capillary LC system coupled to a Q-TOF Micro mass spectrometer (Waters Corporation, Milford, MA, USA). The nanoflow ESI source conditions were set as follows: capillary voltage $3500 \mathrm{~V}$, sample cone voltage $35 \mathrm{~V}$, extraction cone voltage $1 \mathrm{~V}$, source temperature $120{ }^{\circ} \mathrm{C}$, cone gas $\left(\mathrm{N}_{2}\right) 10 \mathrm{~L} / \mathrm{h}$. Chromatographic separations were performed on a homemade C18 reversed phase capillary column $(75 \mu \mathrm{m}$ internal diameter, $150 \mathrm{~mm}$ length, $3 \mu \mathrm{m}$ particle size). The mobile phases used were: deionized water with $0.1 \%$ formic acid (A); acetonitrile with $0.1 \%$ formic acid (B). The reconstituted extract was injected and loaded onto the trap column (Zorbax 300SB-C18 Nano trapping column, Agilent Technologies, Santa Clara, CA, USA) using $95 \%$ mobile phase A and $5 \%$ mobile phase B at a flow rate of $10 \mu \mathrm{L} / \mathrm{min}$ for $10 \mathrm{~min}$. Following this, the stream select module was switched to align the trap column with the analytical column, and a linear gradient of mobile phases A and B was carried out, progressing from $5 \%$ to $45 \%$ over $60 \mathrm{~min}$. A data dependent acquisition was employed for the MS survey scan and the selection of three precursor ions and subsequent MS/MS of the selected parent ions. The MS scan range was from $m / z 400-1800$ and the MS/MS scan was from $m / z 50-1800$. The MS/MS de novo sequencing was performed with a combination of manual sequencing and automatic sequencing by PepSeq software (Waters Corp., Milford, MA, USA).

\section{Data Analysis}

Briefly, the peak lists of mass spectra from 4800 MALDI TOF/TOF were exported from the Data Explorer software, and pasted into Microsoft Excel. The peak lists were compared with an in-house database of crustacean neuropeptides and filtered manually for known neuropeptide peaks. In this home-built neuropeptide database, we included both our own de novo sequencing results and neuropeptide sequences reported by other groups. Specifically, tblastn (http://www.ncbi.nlm.nih.gov/BLAST/) was used to mine for expressed sequence tags (ESTs) encoding putative $C$. sapidus peptide precursors (taxid: 6763) via queries using known arthropod preprohormone sequences. The hits were translated using the Translate tool of ExPASy (http:// www.expasy.ch/tools/dna.html). The translated preprohormone sequences were used to predict mature peptide sequences and assist our de novo sequencing process. The presence or absence of a peak was determined from each spectrum using $30 \mathrm{ppm}$ as mass error tolerance. The peak list of mass spectra from SolariX MALDI-FTMS was processed by the flexImaging software, compared to an inhouse database of crustacean neuropeptides, and filtered manually for known neuropeptide peaks with a mass error tolerance of $3 \mathrm{ppm}$.

\section{Results and Discussion}

\section{Direct Tissue Profiling of Neuropeptides in C. sapidus STNS}

Characterization of neuropeptides in the STNS is inherently challenging because of the low pico- to sub-femtomolar in vivo concentrations of neuropeptides expressed in such small neural tissue [45-47]. The STNS lies between the brain and the suboesophageal ganglion (SOG) and consists of four ganglia together with connecting and motor nerves as shown in Figure 1a. The ganglia are the paired CoGs, OG, and STG. The OG lies between the two CoGs and is connected with them by the inferior esophageal nerves (ion). The STG is also connected with the CoGs by the superior esophageal nerves (son) and the stn. The STG is located within the ophthalmic artery and the substances released by the neurohemal organs, the pericardial organs (PO), are transported to the STG via the artery by the heart. The STNS freshly dissected out of a blue crab C. sapidus and subjected to subsequent preparation for profiling and imaging experiments is shown in Figure 1b. A black sand paper was added beneath the STG as background for increased contrast during optical image scanning.

Direct tissue analysis of these ganglia provides a snapshot of the neuropeptides present in the tissues. However, the 


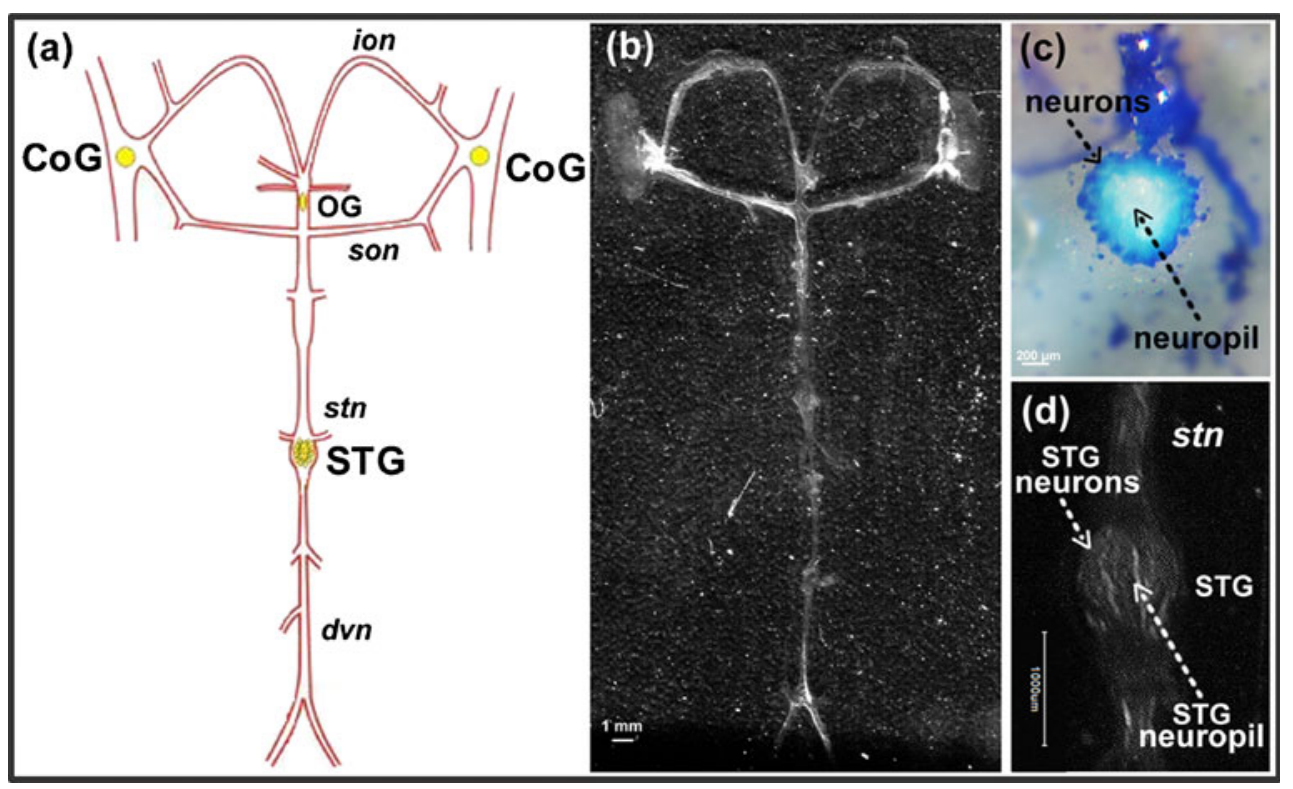

Figure 1. Images of $C$. sapidus STNS and STG. (a) A schematic of the C. sapidus STNS, with the major ganglia and nerves labeled (figure courtesy Dr. Nadim and Dr. Marder labs). (b) An optical image of the isolated C. sapidus STNS mounted on a glass slide and subjected to subsequent IMS experiments. (c) An optical micrograph of a desheathed STG that clearly revealed its neuropil and surrounding motorneurons after methylene blue staining. (d) An optical image of the isolated STG mounted on a glass slide under microscope. The corresponding scale bar is annotated in each panel

tube-like sheath wrapping around the ganglion significantly hindered the effective detection of neuropeptides. To improve the sensitivity, we removed the sheath around the ganglia in the STNS. In Figure 1c, the center neuropil and the surrounding methylene blue-stained motorneurons are easily visualized under microscope after removing the sheath around the STG. Moreover, the neuropeptides are extracted more efficiently out of the ganglion by matrix solution, facilitating tissue profiling.

Figure $2 \mathrm{a}, \mathrm{b}$, and $\mathrm{c}$ show representative annotated mass spectra collected by direct tissue analysis from the CoGs, STG and stn on MALDI-TOF/TOF, respectively. Peptides belonging to 10 different families were detected, including A-type and B-type allatostatins (AST), RFamide, orcokinin, tachykinin-related peptide (TRP), SIFamide, crustacean hyperglycemic hormone precursor related peptides (CPRP), pyrokinin, RYamide, and YRamide. Distinct neuropeptide profiles were observed at different locations of the STNS, including both qualitative and relative quantitative differences. For instance, Cancer borealis TRP (CabTRP) 1a APSGFLGMRa $(\mathrm{m} / \mathrm{z}$ 934.49) was detected as one of the most abundant peptides at all three locations; however, it only dominated the profiling spectrum of CoGs. Its isoform YPSGFLGMRa $(\mathrm{m} / \mathrm{z}$ 1026.52), a recently discovered Callinectes sapidus TRP (CalsTRP), was also more concentrated in the CoGs than the STG or stn. The expression of TRP families in the STG, stn and $\mathrm{CoG}$ is in agreement with previous immunohistochemical studies visualizing their presence in the STNS of Cancer borealis. Moreover, both TRPs were reported to regulate feeding behavior in the crab Callinectes sapidus by exciting the gastric mill (food chewing) and pyloric (food filtering) rhythm [48]. An YRamide, HIGSLYRa $(m / z$ 844.48), was the second dominant peptide in the CoGs, whereas it exhibited relatively the same peak intensity as the other abundant RFamide and orcokinin peptides in the STG profiling spectrum. A SIFamide, GYRKPPFNGSIFa $(\mathrm{m} / \mathrm{z}$ 1381.74), also displayed a similar trend as the YRamide in terms of relative intensity among the three locations. Interestingly, the RYamide family, including SGFYANRYa ( $\mathrm{m} / z$ 976.46) and pEGFYSQRYa ( $m / z$ 1030.45), was detected exclusively in the CoGs, suggesting their significantly higher abundance in CoGs than STG. In contrast, B-type AST (AST-B), RFamide and orcokinin families were generally detected with higher signal intensities in the STG and stn than in the CoG, such as the AST-B peptides, AGWSSMRGAWa ( $m / z$ 1107.52), SGDWSSLRGAWa ( $\mathrm{m} / \mathrm{z}$ 1220.58), and VPNDWAHFRGSWa $(\mathrm{m} / \mathrm{z}$ 1470.70). Nevertheless, exceptions were noticed; the orcokinin peptides NFDEIDRSGFGFA $(\mathrm{m} / \mathrm{z} 1474.66)$ and NFDEIDRSSFGFA $(\mathrm{m} / \mathrm{z}$ 1504.67) were more abundant in the STG, whereas the orcokinin NFDEIDRSSFGFN $(\mathrm{m} / \mathrm{z}$ 1547.68) exhibited a significantly higher expression level than the other RFamides, AST-Bs or orcokinins in the CoGs. In contrast to AST-B, another family belonging to allatostatin, A-type allatostatin (AST-A) neuropeptides were detected with relatively low abundance. In addition, none of C-type ASTs (AST-C) were observed in this study, probably because of their low expression levels in $C$. sapidus STNS. The differential expression patterns of neuropeptides in the STNS demonstrate the complex structure of crustacean STNS and the heterogeneous distributions of multiple neuropeptides, indicating diverse roles of neuropeptides played in various physiological activities. 

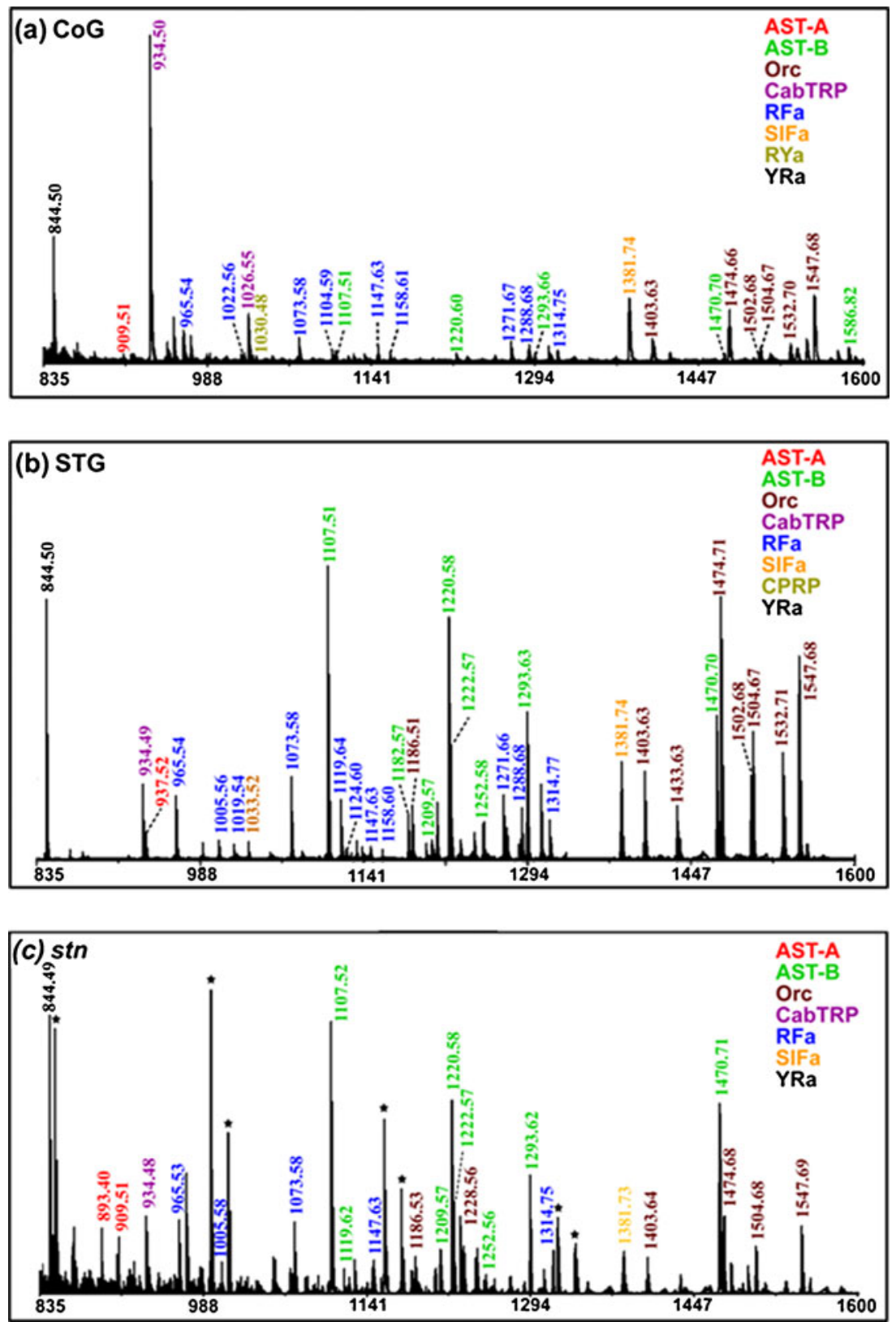

Figure 2. Comparison of direct tissue mass spectra from three discrete locations of $C$. sapidus STNS, including (a) CoG, (b) STG, and (c) stn. Peptides are annotated with molecular weight and their corresponding families. Note that not all neuropeptides detected via direct tissue analysis are evident in these representative spectra. The peaks labeled with asterisks are assigned as interferents

Other than the STG, CoGs, and stn, other neural organs and interconnecting nerves were also characterized. Nevertheless, only a few neuropeptides were detected in the OG, probably because of the relatively low abundance of neuropeptides expressed in the OG, and fewer neurons and much smaller tissue size. The nerves son that connect STG with $\mathrm{CoG}$ and the nerves ion that connect CoGs with OG were also analyzed. Very limited intensity of signals arose from either son or ion (data not shown).

Although MALDI-TOF/TOF is capable of generating quick profiles of neuropeptides directly from tissue samples and even single cells, the high mass measurement accuracy, and high mass resolving power achievable with FTMSbased instrumentation enables identification of neuropeptides from the complex neurosecretory organs and nerves in the crustacean model systems with improved confidence. Herein, a high performance 12 Tesla MALDI-FT-ICR was employed to analyze neuropeptides expressed in the STG. The high quality MS data complemented and confirmed the MALDI-TOF/TOF results as detailed in Supplemental Table 1. For example, the putative neuropeptide peak at $\mathrm{m} / \mathrm{z}$ 1019.54 cannot be assigned to the known neuropeptide 
APRNFLRFamide $(\mathrm{m} / \mathrm{z}$ 1019.59) based on a mass error tolerance of $30 \mathrm{ppm}$ by mass matching with an in house $C$. sapidus neuropeptide database. However, the ultra-high resolving power of FT-ICR realized the separation of two ions with a 0.09 Da mass difference and detected two peaks at $m / z$ of 1019.50 and $1019.59 \mathrm{Da}$, respectively. Therefore, the peak detected at $\mathrm{m} / \mathrm{z} 1019.54$ on MALDI-TOF/TOF could be attributed to an unresolved peak containing two molecular species that could only be unambiguously distinguished on an ultra-high performance mass analyzer. The utilization of FT-ICR can resolve closely related masses from a highly complex neural tissue; moreover, it provides the requisite specificity for elucidating molecular species without a sacrifice in sensitivity.

A number of peptide peaks were detected on both instruments, whereas some were uniquely identified by one of the instruments, because of the preferential detection of certain peptide ions with different mass analyzers. For example, the peptide GSNWSNLRGAWamide $(\mathrm{m} / \mathrm{z}$ 1246.61) was detected on FT-ICR rather than TOF/TOF, whereas the TRP peptides, CabTRP 1a and CalsTRP, were not observed on FT-ICR but showed at relatively high intensity on $\mathrm{TOF} / \mathrm{TOF}$.

Peptide identification is greatly facilitated by the accurate mass measurements provided by FT-ICR. In addition, the neuropeptidome of other neural organs of $C$. sapidus has been characterized, providing a reservoir of potential neuropeptides that might be present in the STNS. Nevertheless, further confirmation by MS/MS fragmentation experiments is desirable for neuropeptide identification based solely upon accurate mass measurements. Supplemental Figure S1a shows the in situ collision-induced-dissociation (CID) MS/MS spectra of an orcokinin peptide NFDEIDRSGFGFA $(\mathrm{m} / \mathrm{z}$ 1474.66) performed directly on the STG. As seen, almost complete series of fragment ions were detected, confirming high fidelity for peptide identification. Figure S1b is another MS/MS spectrum of a peptide at $\mathrm{m} / \mathrm{z}$ 844.50. Based on its accurate mass and known sequence, the sequence was confirmed as HIGSLYRa. It is worth mentioning that the Ile/Leu ambiguity resulting from the isobaric nature of these amino acids has been identified through EST homology search from Carcinus maenas [49]. Intriguingly, several signature peaks typically belonging to lipid species were also observed in the spectrum, such as neutral loss of trimethylamine group $\triangle 59 \mathrm{Da}$, product ion from the phosphocholine head chain $(\mathrm{m} / \mathrm{z} 184.08)$, and fragments from choline $(\mathrm{m} / \mathrm{z} 86.13$ and 104.13). The accurate mass and the characteristic fragmentation pattern of phosphatidylcholine (PC) species allow the molecule to be sequenced as a $\mathrm{K}^{+}$adduct form of PC (38:6). The inclusion of the PC lipid at such a close mass $(\mathrm{m} / \mathrm{z}$ 844.53) during direct tissue analysis also explains the detected mass to be 844.50 Da rather than the theoretical value of YRamide ( $m / z$ 844.48) alone. In comparison to in situ MS/MS performed on TOF/TOF, biochemical separation coupled to MS/ MS sequencing via nanoLC-ESI-Q-TOF MS/MS was commonly exploited to confirm the identity of the known neuropeptides and to de novo sequence novel neuropeptides. Supplemental
Figure S2 shows two examples of MS/MS spectra acquired on ESI-Q-TOF, including CabTRP $1 \mathrm{a}(\mathrm{m} / \mathrm{z}$ 934.49, Figure S2a) and a SIFamide peptide $(\mathrm{m} / \mathrm{z}$ 1381.74, Figure S2b). This classic approach combined with in situ MS/MS sequencing and direct tissue profiling resulted in the identification of 55 peptides from 10 families, including 50 neuropeptides discovered in other neural organs of $C$. sapidus like CalsTRP and five peptides previously identified in other species, but new to the $C$. sapidus species. Collectively, this work demonstrated the advantages of implementing combined MALDI-TOF/TOF and MALDI-FT-ICR platforms for enhanced sensitivity, speed, and high mass resolution, for direct tissue profiling. The identification based on accurate mass matching and limited in-situ MS/MS via MALDI platform is complemented and validated by the employment of nanoLC-ESI tandem MS de novo peptide sequencing. The comprehensive characterization and mapping of various neuropeptides from $C$. sapidus STNS will provide strong framework for future physiological investigations of neuropeptides in crustacean STNS.

\section{High Spatial Resolution Imaging of Neuropeptides in C. sapidus STG by MALDI-TOF/TOF}

Removal of the sheath from the STG is shown to improve the extraction and detection of neuropeptides for direct tissue analysis. Nevertheless, existence of the sheath maintains the integrity and intactness of the tissue as shown in Figure 1d, hindering the release of neuropeptides from the ganglion and preventing severe analyte diffusion during matrix application in subsequent IMS experiments. Consequently, sufficient extraction of the peptides from intact STG was achieved after multiple matrix coating. MS images that can be accurately co-registered with the optical image were obtained with intact STG, yet most neuropeptides distributed outside the edge of STG after matrix coating without the sheath. Hence, the sheath is kept intact in the application of STG IMS. The humidity of matrix application was adjusted accordingly to achieve maximal neuropeptide extraction out of the sheath while retaining their original localization.

A wealth of biochemical information is provided in a single MALDI-TOF/TOF IMS measurement and various neuropeptides exhibit significantly different or similar spatial distribution patterns. The utilization of autoflex III MALDI-TOF/TOF equipped with a highly focused laser smartbeam allows for acquisition of multiple high resolution neuropeptide images from the STG using $25 \mu \mathrm{m}$ as step size. Images of most neuropeptide isoforms detected in direct tissue analysis were acquired, consuming merely $85 \mathrm{~min}$ of instrument time. Figure 3a shows the optical image of a $C$. sapidus STG. Representative MS images of the neuropeptides, including CabTRP 1a, three AST-Bs, three RFamides, a SIFamide, and an orcokinin, are shown in Figure $3 \mathrm{~b}-\mathrm{k}$. All of the neuropeptides were localized within 


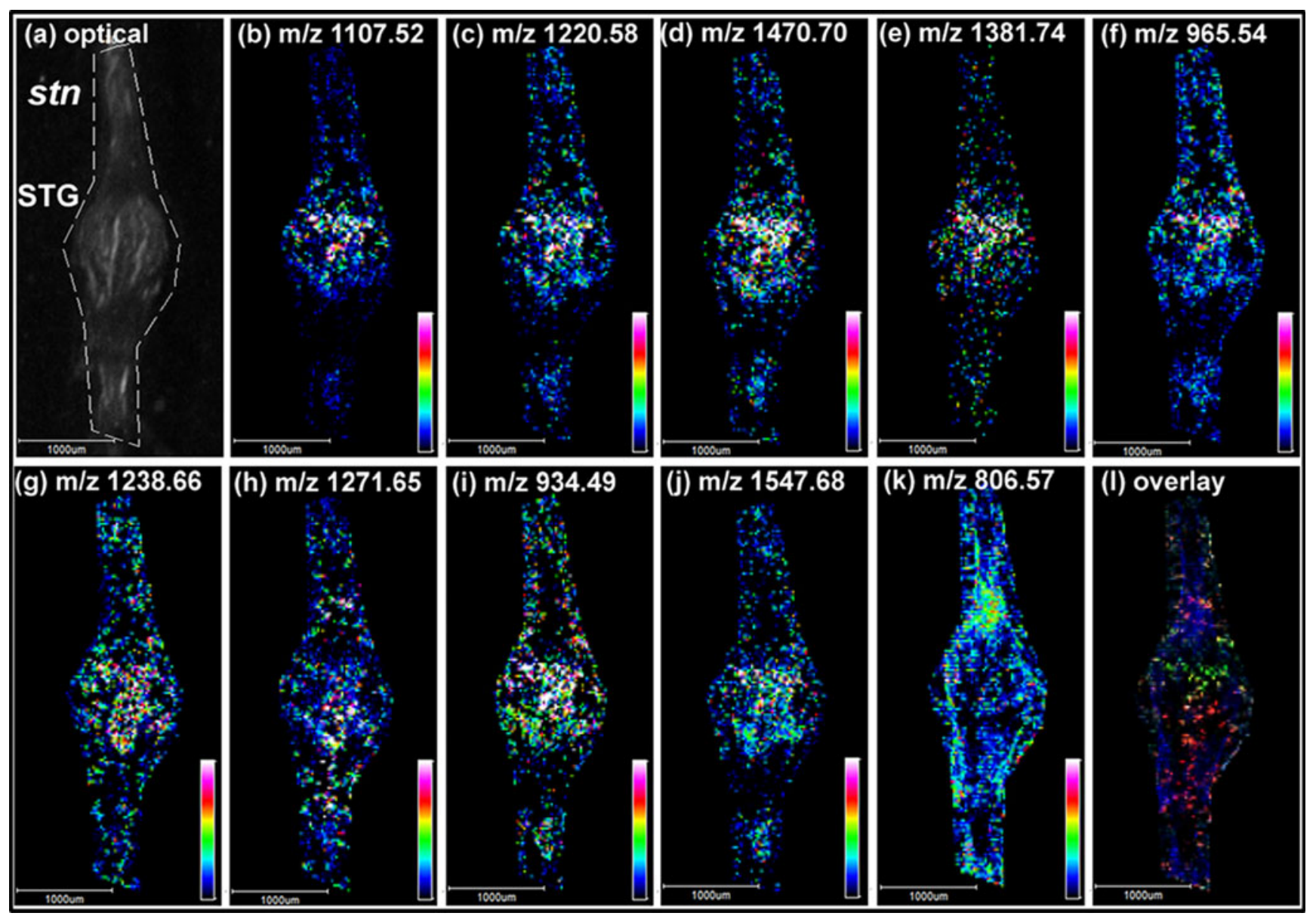

Figure 3. Distribution of neuropeptides in C. sapidus STG revealed by IMS on MALDI TOF/TOF. (a) An optical image of the STG subjected to subsequent IMS preparation. Nine neuropeptides are shown, including three B-type ASTs: (b) AGWSSMRGAWa ( $\mathrm{m} / \mathrm{z}$ 1107.52), (c) SGDWSSLRGAWa ( $\mathrm{m} / \mathrm{z}$ 1220.58), and (d) VPNDWAHFRGSWa $(\mathrm{m} / \mathrm{z}$ 1470.70); (e) SIFamide GYRKPPFNGSIFa ( $\mathrm{m} / \mathrm{z}$ 1381.74); three RFamides: (f) NRNFLRFa $(\mathrm{m} / \mathrm{z}$ 965.54), (g) SQPSKNYLRFa $(\mathrm{m} / \mathrm{z} 1238.66)$, and (h) pQDLDHVFLRFa $(\mathrm{m} / \mathrm{z}$ 1271.64); (i) CabTRP 1a APSGFLGMRa ( $\mathrm{m} / \mathrm{z}$ 934.49); (j) orcokinin NFDEIDRSSFGFN ( $\mathrm{m} / \mathrm{z}$ 1547.68). In addition, the distribution of (k) a lipid PC (38:6) $(\mathrm{m} / \mathrm{z}$ 806.57) is shown. The observed masses are labeled in white above the MS images. Different distribution patterns are shown between different families and also isoforms within individual families; (I) is an overlaid image of (c), (h), and (k), displayed in green, red, and blue, respectively

the STG tissue as shown in the optical image Figure 3a, suggesting negligible analyte diffusion because of the protection from the sheath. As seen in Figure 2b, the AST family dominated the profiling spectrum of the STG. The ASTs are neuropeptides that have important neuromodulatory roles in arthropods. Specifically, AST-B peptides are known for their myoinhibitory role and have been shown to regulate neuronal function in the STNS of $C$. borealis, such as inhibiting the pyloric rhythm in the STG [12, 50]. Most AST-B neuropeptides, such as AGWSSMRGAWa $(\mathrm{m} / \mathrm{z}$ 1107.52), SGDWSSLRGAWa $(\mathrm{m} / z$ 1220.58), and VPNDWAHFRGSWa $(\mathrm{m} / \mathrm{z}$ 1470.70), are expressed in high abundance in the bulbous structure of the neuropil which contains a large number of descending projection fibers and processes from upper modulatory ganglia. Moreover, the relative intensities of the three AST-B peptides (Figure 3bd) appear to be higher at the end towards stn. This is consistent with the fact that the AST-B peptides are detected with high intensity in stn shown in Figure 2c but not detectable in the nerve extended posteriorly toward the $d v n$ (data not shown). A recent immunohistochemical study of VPNDWAHFRGSWa confirmed our observation. No immunoreactivity to VPNDWAHFRGSWa (named CbAST-B1 in that report) was detected in the STG somata, yet extensive staining in the STG neuropil and the fibers that projected from the stn into the neuropil was observed [12]. The antibody-based staining is capable to achieve high spatial resolution mapping of single cell and fibers. However, it provides fairly limited chemical information and lacks specificity to determine the distribution of the different isoforms among AST-Bs or possibly all the AST family of peptides, even assuming the antibody employed is likely to have a higher affinity for the CbAST-B1. The utilization of IMS can generate images for each AST-B isoform unambiguously and combine these images to produce multiplexed images useful for determining peptide isoform co-localiza- 
tion and distinct distribution. In this case, the three AST-B peptides have similar structure and show colocalization within the STG, suggesting that the AST-B receptor might not distinguish among these peptides. Besides AST-Bs, the SIFamide peptide GYRKPPFNGSIFamide shows similar expression pattern in the STG neuropil as shown in Figure 3e. This Gly ${ }^{1}$-SIFamide has been broadly reported in nervous system of crustacea, such as the giant tiger prawn Penaeus monodon [51], Jonah crab Cancer borealis [52] and the crayfish family [53]. Nevertheless, immunohistochemical staining results have only been reported for $\mathrm{Val}^{1}$-SIFamide in the STNS but not for Gly ${ }^{1}$-SIFamide. Our work for the first time reported the localization of this specific SIFamide among many other neuropeptides, filling the gap for neurobiologists. Moreover, the coexistence of the peptides from two families possibly implies their synergistic interactions in crustacean STNS biological circuits. Other than AST-Bs, most RFamides were also expressed in high abundance in the STG and localized in a family colocalization pattern, although exceptions exist. As shown in Figure $3 \mathrm{f}-\mathrm{h}$, distribution of NRNFLRFa $\left(m / z\right.$ 965.54) resembled the AST-Bs and Gly ${ }^{1}$ SIFamide, whereas SQPSKNYLRFa $(\mathrm{m} / z$ 1238.66) was localized more evenly within the STG neuropil, with pQDLDHVFLRFa $(\mathrm{m} / \mathrm{z}$ 1271.64) being concentrated in the neuropil but more at the end close to $d v n$. This phenomenon suggests that neuropeptides belonging to the same family, yet with slightly different sequences, could display varied localizations within the STNS, probably indicating their distinct biological functions. Clearly, IMS technique serves as the most suitable tool to examine the individual members from a complex composition of each neuropeptide family, facilitating the elucidation of possible distinct actions of various peptide isoforms within a superfamily. CabTRP 1 a $(\mathrm{m} / \mathrm{z}$ 934.49) in Figure $3 \mathrm{i}$ and the orcokinin peptide NFDEIDRSSFGFN $(\mathrm{m} / \mathrm{z}$ 1547.68) in Figure $3 j$ were both evenly distributed within the STG neuropil region and appear to co-localize. Both peptides were expressed in high abundances in the STG, yet their distributions in the STG were examined for the first time in this IMS study. Despite the ultra-high resolution images provided by immunohistochemical staining (IHC), IMS complements this technique by enabling the mapping of multiple neuropeptides unambiguously with no prior knowledge at a spatial resolution that is high enough to differentiate various peptides within such a small organ, of which the length is around $1 \mathrm{~mm}$ and the width is less than $0.5 \mathrm{~mm}$. Moreover, current advancements in reducing laser beam size will facilitate future applications of neuropeptide imaging at cellular level [34].

Lipids were previously considered as interfering species for neuropeptide detection; however, increased interest has been drawn to understand potential roles of lipids present in the nervous system. Figure $3 \mathrm{k}$ shows the MS image of a lipid $(\mathrm{m} / \mathrm{z}$ 806.57) that displays significantly higher intensity on the nerves and the sheath than that in the STG neuropil. This observation agrees well with previous knowledge that the sheath enclosing axons in invertebrates displayed remarkable similarities with vertebrate myelin sheath in terms of ultra- structure [54]. As expected, lipids distributed mostly in this whitish, fatty layer rather than the neuropil. This observation illustrates a clearly distinct overall spatial localization patterns from the majority of neuropeptides. Using the overlay function of the imaging software, a superimposed MS image of various molecular species, including the AST-B neuropeptide at $\mathrm{m} / \mathrm{z}$ 1220.58 false-colored in red, the RFamide peptide of $\mathrm{m} / \mathrm{z}$ 1271.64 coded in green, and the lipid PC(38:6) shown in blue, is obtained, displaying the distinct distribution between neuropeptides and lipids within such a small ganglion. This intriguing observation implicates potentially different functions of the differentially-localized neuropeptides in the STG as well as the distinction between the peptides and lipids in the $C$. sapidus nervous system. The application of IMS at high spatial resolution offers a new level of understanding of the STNS by these well-resolved images of multiple molecular species and co-localization patterns of potentially interacting molecular players.

\section{High Mass Spectral Resolution Imaging of Neuropeptides in C. sapidus STG by MALDI-FT-ICR}

High spatial resolution MS images are generated by TOF/ TOF instruments rapidly, but these analyzers suffer from modest mass resolving power and mass accuracies. FT-ICR mass analyzer can provide comparable spatial resolutions to a TOF/TOF analyzer while offering superior resolution and accuracy for unequivocal assignment of analytes [55]. Therefore, MALDI-FT-ICR has herein been applied to MALDI IMS of the STG as a great alternative to TOFbased tissue imaging experiments. Figure 4 demonstrates the essence of utilizing high-mass-accuracy and high-massresolution FT-ICR for neuropeptide imaging. Figure $4 \mathrm{a}$ is an $\mathrm{m} / \mathrm{z}$ 1000-1500 spectrum averaging data acquired throughout the entire STG, whereas insets Figure $4 \mathrm{~b}$ and c show two magnified mass ranges. In Figure $4 b$, the two different compounds at $\mathrm{m} / \mathrm{z} 1019.50$ and 1019.59 are resolved unequivocally, revealing the high resolution and quality of the FT-ICR-based IMS data. Moreover, these two species exhibit a different spatial distribution in the STG as shown in the images in Figure 4d-e. The lower-abundance peak of $\mathrm{m} / \mathrm{z} 1019.50$ was concentrated in the $s t n$ area as shown in Figure 4d, and the peak of $m / z 1019.59$ with higher signal intensity distributed mainly in the STG neuropil as displayed in Figure 4e. The overlaid image of these two compounds clearly demonstrated their distinct locations by false-color coding in red and green, respectively. Based on the accurate mass obtained on FT-ICR and its concentrated distribution in the STG neuropil, the peak at $\mathrm{m} / \mathrm{z} 1019.59$ was assigned as the neuropeptide APRNFLRFamide with high confidence based on a $0.3 \mathrm{mDa}$ mass error. In contrast, the modest mass spectral resolution of TOF/TOF cannot resolve the two compounds at 1019.50 and $1019.59 \mathrm{Da}$ and, thus, the discrimination cannot be made, resulting in an image combining the spatial distribution of these two 
compounds. The mass accuracy is also compromised by the lower spectral resolution of TOF/TOF, generating an unresolved peak at $\mathrm{m} / \mathrm{z} 1019.54$ in Figure 2b. Hence, the neuropeptide cannot be identified on a TOF platform, given that the mass error tolerance for neuropeptide assignment by mass measurement was set at $30 \mathrm{ppm}$. Another example involved the two peaks at $\mathrm{m} / \mathrm{z} 1474.66$ and 1474.74 that were separated in this very narrow mass range shown in
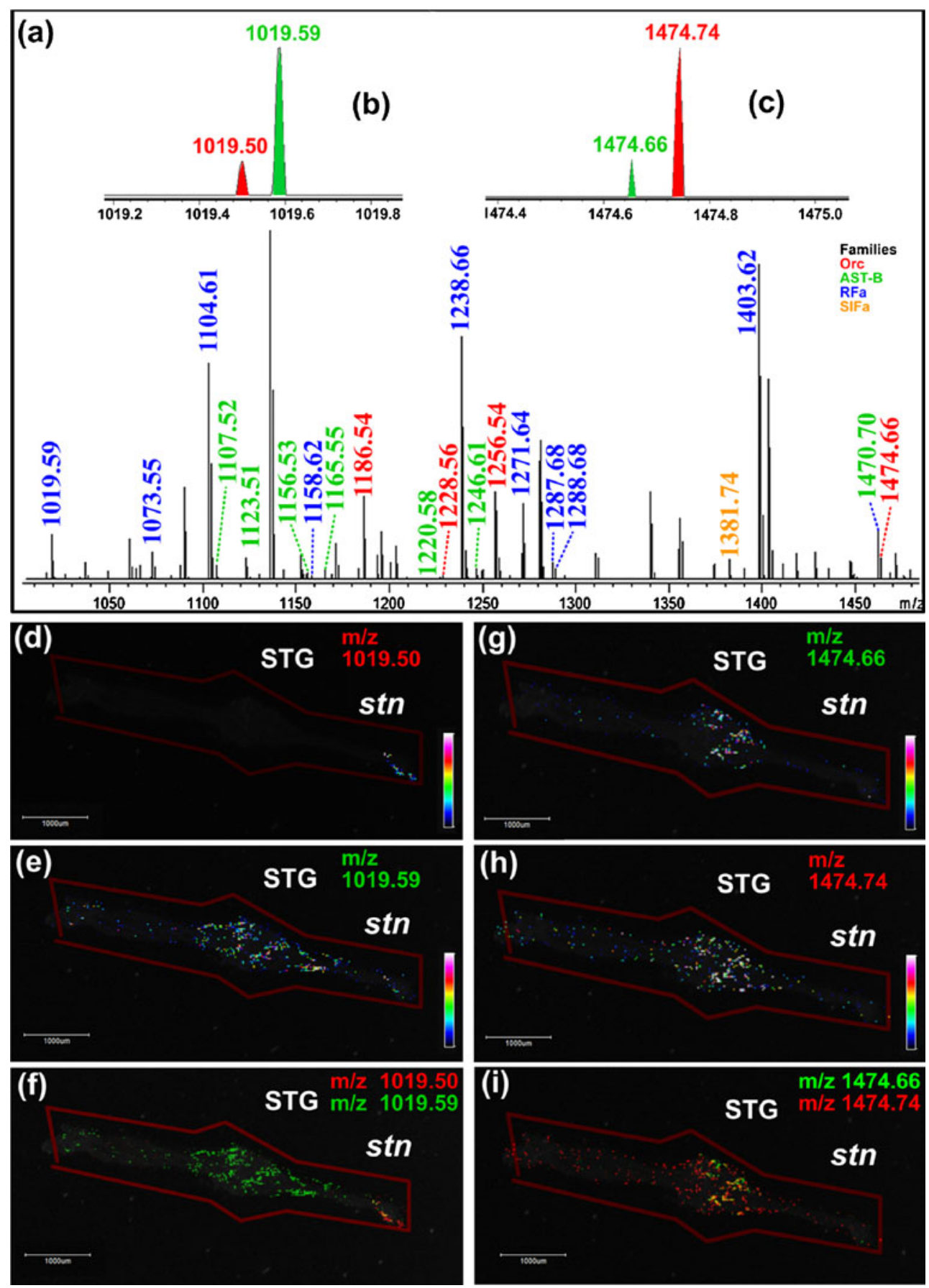

Figure 4. Localization of neuropeptides in C. sapidus STG revealed by IMS on MALDI-FT-ICR. (a) Total MALDI-FT-ICR mass spectrum of the $C$. sapidus STG. Peptides are annotated with molecular weight and color-coded according to their corresponding families; (b) and (c) are two inserts showing the zoom-in spectra of two ion clusters $\mathrm{m} / \mathrm{z}$ 1019-1020 and 1474-1475; (d) and (e) show the distribution of the ions at $\mathrm{m} / \mathrm{z} 1019.50$ and 1019.59; (f) shows an overlaid image of (d) and (e), visualizing the different distribution of the $\mathrm{m} / \mathrm{z} 1019.50$ ion displayed in green and the $\mathrm{m} / \mathrm{z} 1019.59$ ion displayed in red. Similarly, (g) and (h) manifests the localization of the peaks at $\mathrm{m} / \mathrm{z} 1474.66$ and 1474.74 , respectively; (i) is a superimposed image of the 1474.66 Da ion displayed in green and the 1474.74 Da ion displayed in red, visually demonstrating their distinct localization in the STG 
Figure $4 \mathrm{c}$. Figure $4 \mathrm{~g}$ shows that the putative peptide of $\mathrm{m} / \mathrm{z}$ 1474.66 was distributed primarily in the STG neuropil and more concentrated at the end close to the stn, whereas the peak at $\mathrm{m} / \mathrm{z} 1474.74$ was documented to be localized in the STG neuropil and also the connecting nerve in Figure $4 \mathrm{~h}$. The peak at $\mathrm{m} / \mathrm{z} 1474.66$ was assigned as an orcokinin peptide NFDEIDRSGFGFA by the mass and the distribution that resembles other orcokinin neuropeptides; nevertheless, the peak at $\mathrm{m} / \mathrm{z} 1474.74$ did not match any sequenced neuropeptides. In Figure 4i, the overlaid FT-ICR-MS image of the orcokinin neuropeptide and the putative peptide displayed overlapping but not identical expression patterns that cannot be differentiated by TOF/TOF. Moreover, the utilization of TOF/TOF resulted in a detected mass of $\mathrm{m} / \mathrm{z}$ 1474.71 as previously shown in Figure 4b, probably because of the coexistence of the two analytes at $\mathrm{m} / \mathrm{z} 1474.66$ and 1474.74, respectively. The orcokinin could have been misassigned as an interference peak due to the $5 \mathrm{mDa}$ mass error. These two examples clearly demonstrate the contribution of high mass resolution and accuracy provided by FTICR to the successful identification of neuropeptides from minute quantities of complex biological samples. Its combination with MALDI-TOF/TOF in the application of neuropeptide IMS provides unequivocal detailed localization information (as low as $25 \mu \mathrm{m}$ lateral resolution) with the most comprehensive coverage of neuropeptides from the crustacean STG to date. This novel multi-faceted MS-based strategy can also be easily adapted to image other neural tissues, making this approach a valuable tool for neuropeptidomics studies.

An inherent characteristic of all FT-based analyses is that achievable mass resolving power is directly proportional to analysis time; hence the greater the desired mass resolving power, the longer one must measure ions in the detector. However, recent gains in FT-ICR technology, including the ability to desorb and accumulate ions externally during the detection event [56] (Áccumulate During Detect) has brought FT-ICR measurement speeds close to those achieved with TOF [56]. As an example, the measurement region shown here consists of 5200 pixels analyzed at a rate of $1.33 \mathrm{~s}$ per pixel, or 45 pixels per min with an achieved mass resolving power of $400,000 \mathrm{~m} / \Delta \mathrm{m}$ at $\mathrm{m} / \mathrm{z} 400$, reflecting a 100 -fold increase in spectral resolution over the TOF measurement (estimated $4000 \mathrm{~m} / \Delta \mathrm{m}$ at $\mathrm{m} / \mathrm{z} 400$ ). This brings the total measurement time for the STG image to $115 \mathrm{~min}$, or just under $2 \mathrm{~h}$ for the 12 Tesla FT-ICR employed in the study, comparable to $85 \mathrm{~min}$ acquisition time on TOF/TOF. While higher magnetic fields can utilize shorter transients without a sacrifice of spectral resolution, similar results could be achieved at lower field strengths with only a marginal increase in total measurement time. It is worth noting that for any given fields strength, spectral resolution can be effectively doubled through application of a recently reported absorption mode phasing as an alternative to traditional magnitude mode signal processing [57].
As for the overlapping yet some distinct peptide detection results obtained from the TOF/TOF and FT-ICR instruments as shown in Supplemental Table 1, this observation is not too surprising and has been previously documented in the studies of neuropeptides via direct tissue analysis [15, 58]. Although in theory, one would expect that the same sample analyzed by two mass analyzers should reveal the same species, in practice the resulting mass spectral profiles can be affected by numerous factors, including the instrumental configuration that impacts the ion transmission efficiency, ion transport time, and the delay time between ion desorption/ionization and detection. Specifically, MALDI TOF/TOF enables fast acquisition and offers sensitive detection of a broad mass range of peptides. The generated ions are quickly transferred and detected upon their formation in the MALDI source. In contrast, MALDI FTICR provides superior resolving power, but the relatively long pulse sequence and the space-charge limitations in the cell could bias the detection of some low-abundance species. The ions produced by the intermediate-pressure MALDI source undergo collisional cooling by nitrogen gas, storage in a hexapole, followed by transfer and analysis in the ICR cell. This leads to the preservation and detection of more stable peptide ions [59] and fewer fragment ions via metastable decay due to superior cooling of the ions with the intermediate pressure source [60]. All the factors discussed above can cause the differences in peptides we observed on two different instrumental platforms. However, the discrepancies can be interpreted as complementarities when results obtained by MALDI-TOF/TOF and MALDI-FT-ICR are combined. The complementarities between the TOF and FT-ICR-based instruments are appreciated by Römpp et al. in a study of tryptic peptides that were differentially expressed in breast cancer patients [61]. da Silva et al. also noticed an increased number of the detected BSA tryptic peptides and an improvement of sequence coverage when evaluating the combination of MALDI-TOF and MALDI-FT-ICR in peptide mass fingerprinting [60].

\section{IMS of C. sapidus STNS}

The STNS of C. sapidus consists of the STG, the OG, and the paired CoGs as well as a number of interconnecting and motor nerves. In the section of STNS profiling, we examined the neuropeptides from different regions of STNS; however, only the STG, CoG, and stn generated neuropeptide-rich mass spectra. Only two orcokinin peptides, NFDEIDRSGFa $(\mathrm{m} / z$ 1198.55) and NFDEIDRSSFa $(\mathrm{m} / z$ 1228.56), and an AST-B peptide VPNDWAHFRGSWa $(\mathrm{m} / \mathrm{z}$ 1470.70), were detected in the OG. Moreover, no other putative neuropeptide peaks were detected from other regions in the STNS, probably because of their relatively low level of neuropeptide expression in the interconnecting nerves. Nevertheless, the first IMS study of neuropeptides from the crustacean STNS still demonstrated the potential of mapping multiple trace-level neuropeptides unambiguously and simultaneous- 
ly from complex neural network. Representative neuropeptide images are shown in Supplemental Figure S3. Figure S3a presents the localization of the YRamide HIGSLYRa $(m / z$ 844.48) that shows higher abundance in the STG and lower abundance in the CoG. This observation is also in agreement with the relative abundance of this neuropeptide observed in the STG and CoG profiling spectra in Figure 2a and $\mathrm{b}$. In Figure S3b, the orcokinin NFDEIDRSSFa $(\mathrm{m} / \mathrm{z}$ 1228.56) is distributed throughout the STNS of C. sapidus, which agrees well with our previous profiling results. Figure $\mathrm{S} 3 \mathrm{c}$ presents the localization of another orcokinin NFDEIDRSSFGFV $(\mathrm{m} / \mathrm{z} 1532.70)$ that is particularly concentrated in the STG and the CoG. Other than neuropeptides, Figure S3d displays a potassiated lipid PC (36:1) at $\mathrm{m} / \mathrm{z}$ 826.57, the abundance of which is higher in connecting nerves than ganglia in comparison to neuropeptides in the STNS. This observation is in agreement with the highspatial-resolution images of the neuropeptides and lipids expressed in the STG as shown in Figure 3.

In summary, this integrative IMS platform presents a sensitive, reliable, and high-throughput analytical tool for simultaneous mapping of signaling peptides and other types of biomolecules like lipids expressed in low abundance from tissue of minute size. Knowledge of the complement and localization of neuropeptides in the C. sapidus STNS was directly obtained without tedious sample preparation step for liquid phase extraction or immunohistochemical techniques. Moreover, the wealth of information gained from IMS of the STNS provides unprecedented molecular details regarding chemical identities and anatomical distributions of these signaling peptides in this classical model nervous system.

\section{Conclusions}

For the first time, MALDI-TOF/TOF and MALDI-FT-ICR were employed for mapping the localizations of neuropeptides at the network and cellular resolution using the $C$. sapidus STNS as a model system. A novel multi-faceted MS strategy which combined direct tissue analysis, in situ MS/ MS and micro-separation coupled to ESI-Q-TOF tandem MS peptide sequencing was employed here. In total, 55 neuropeptides were characterized from $C$. sapidus STNS. The central component of the STNS, STG was imaged for neuropeptide distribution with both TOF/TOF and FT-ICR at a spatial resolution of $25 \mu \mathrm{m}$, generating detailed distribution maps of multiple neuropeptides and lipids simultaneously from this ganglion of minute size. The advantages of FT-ICR added to this platform were manifested by the well-resolved MS images of neuropeptides that could not be distinguished on TOF/TOF. The mass spectral imaging of small STG by FT-ICR combined with imaging of the STNS by TOF/TOF makes the approach a great tool to map neuropeptides in a high-throughput fashion with detailed information gained for the center of the nervous system. In addition, IMS has been applied to image the entire STNS using TOF/TOF because of its fast analysis speed. MS/MS analysis of neuropeptides further elucidated the peptide sequences, rendering a comprehensive study of the complement and localization of neuropeptides in crustacean STNS. The multi-faceted MS-based platform established in this study will benefit the field of neuropeptide imaging and facilitate future investigations on the interplay of a myriad of signaling peptides and neurotransmitters in a neuronal network.

\section{Acknowledgments}

The authors acknowledge support in part for this work by the National Science Foundation grant (CHE-0967784), and National Institutes of Health grants (1R01DK071801, 1R56DK071801). They thank Bruker Daltonics for graciously loaning the Autoflex III MALDI TOF/TOF mass spectrometer. L.L. acknowledges an H.I. Romnes Faculty Research Fellowship.

\section{References}

1. Marder, E., Bucher, D.: Central pattern generators and the control of rhythmic movements. Curr. Biol. 11, R986-R996 (2001)

2. Stemmler, E.A., Peguero, B., Bruns, E.A., Dickinson, P.S., Christie, A.E.: Identification, physiological actions, and distribution of TPSGFLGMRamide: a novel tachykinin-related peptide from the midgut and stomatogastric nervous system of Cancer crabs. J. Neurochem. 101, 1351-1366 (2007)

3. Christie, A.E., Stemmler, E.A., Peguero, B., Messinger, D.I., Provencher, H.L., Scheerlinck, P., Hsu, Y.W.A., Guiney, M.E., de la Iglesia, H.O., Dickinson, P.S.: Identification, physiological actions, and distribution of VYRKPPFNGSIFamide (Val(1)-SIFamide) in the stomatogastric nervous system of the American lobster Homarus americanus. J. Comp. Neurol. 496, 406-421 (2006)

4. Skiebe, P., Dreger, M., Meseke, M., Evers, J.F., Hucho, F.: Identification of orcokinins in single neurons in the stomatogastric nervous system of the crayfish, Cherax destructor. J. Comp. Neurol. 444, 245259 (2002)

5. Nusbaum, M.P.: Regulating peptidergic modulation of rhythmically active neural circuits. Brain. Behav. Evolut. 60, 378-387 (2002)

6. Nusbaum, M.P., Beenhakker, M.P.: A small-systems approach to motor pattern generation. Nature 417, 343-350 (2002)

7. Marder, E.: Motor pattern generation. Curr. Opin. Neurobiol. 10, 691698 (2000)

8. Marder, E., Calabrese, R.L.: Principles of rhythmic motor pattern generation. Physiol. Rev. 76, 687-717 (1996)

9. Dickinson, P.S., Mecsas, C., Marder, E.: Neuropeptide fusion of 2 motor-pattern generator circuits. Nature 344, 155-158 (1990)

10. Marder, E., Bucher, D.: Understanding circuit dynamics using the stomatogastric nervous system of lobsters and crabs. Annu. Rev. Physiol. 69, 291-316 (2007)

11. Sahley, C.L.: Neural integration. Science 260, 244 (1993)

12. Szabo, T.M., Chen, R.B., Goeritz, M.L., Maloney, R.T., Tang, L.S., Li, L.J., Marder, E.: Distribution and physiological effects of B-type Allatostatins (myoinhibitory peptides, MIPs) in the stomatogastric nervous system of the crab Cancer borealis. J. Comp. Neurol. 519, 2658-2676 (2011)

13. Li, C., Kim, K.: Neuropeptides. WormBook, (2009)

14. Nassel, D.R.: Neuropeptides in the nervous system of Drosophila and other insects: multiple roles as neuromodulators and neurohormones. Prog. Neurobiol. 68, 1-84 (2002)

15. Ma, M., Chen, R., Sousa, G.L., Bors, E.K., Kwiatkowski, M.A., Goiney, C.C., Goy, M.F., Christie, A.E., Li, L.: Mass spectral characterization of peptide transmitters/hormones in the nervous system and neuroendocrine organs of the American lobster Homarus americanus. Gen. Comp. Endocrinol. 156, 395-409 (2008) 
16. Ma, M.M., Wang, J.H., Chen, R.B., Li, L.J.: Expanding the crustacean neuropeptidome using a multifaceted mass spectrometric approach. $J$. Proteome Res. 8, 2426-2437 (2009)

17. Kutz, K.K., Schmidt, J.J., Li, L.J.: In situ tissue analysis of neuropeptides by MALDI FTMS in-cell accumulation. Anal. Chem. 76, 5630-5640 (2004)

18. Stemmler, E.A., Cashman, C.R., Messinger, D.I., Gardner, N.P., Dickinson, P.S., Christie, A.E.: High-mass-resolution direct-tissue MALDI-FTMS reveals broad conservation of three neuropeptides (APSGFLGMRamide, GYRKPPFNGSIFamide and pQDLDHVFLRFamide) across members of seven decapod crustacean infraorders. Peptides 28, 2104-2115 (2007)

19. Stemmler, E.A., Provencher, H.L., Guiney, M.E., Gardner, N.P., Dickinson, P.S.: Matrix-assisted laser desorption/ionization Fourier transform mass spectrometry for the identification of orcokinin neuropeptides in crustaceans using metastable decay and sustained offresonance irradiation. Anal. Chem. 77, 3594-3606 (2005)

20. Defelipe, J.: Neocortical neuronal diversity-chemical heterogeneity revealed by colocalization studies of classic neurotransmitters, neuropeptides, calcium-binding proteins, and cell-surface molecules. Cereb. Cortex 3, 273-289 (1993)

21. Skiebe, P.: Neuropeptides in the crayfish stomatogastric nervous system. Microsc. Res. Techniq. 60, 302-312 (2003)

22. Ye, H., Greer, T., Li, L.J.: From pixel to voxel: a deeper view of biological tissue by 3D mass spectral imaging. Bioanalysis 3, 313-332 (2011)

23. Nemes, P., Vertes, A.: Laser ablation electrospray ionization for atmospheric pressure, in vivo, and imaging mass spectrometry. Anal. Chem. 79, 8098-8106 (2007)

24. Li, Y., Shrestha, B., Vertes, A.: Atmospheric pressure infrared MALDI imaging mass spectrometry for plant metabolomics. Anal. Chem. 80, 407-420 (2008)

25. Wiseman, J.M., Ifa, D.R., Song, Q.Y., Cooks, R.G.: Tissue imaging at atmospheric pressure using desorption electrospray ionization (DESI) mass spectrometry. Angew. Chem. Int. Ed. 45, 7188-7192 (2006)

26. Galhena, A.S., Harris, G.A., Nyadong, L., Murray, K.K., Fernandez, F.M.: Small molecule ambient mass spectrometry imaging by infrared laser ablation metastable-induced chemical ionization. Anal. Chem. 82, 2178-2181 (2010)

27. Stoeckli, M., Chaurand, P., Hallahan, D.E., Caprioli, R.M.: Imaging mass spectrometry: a new technology for the analysis of protein expression in mammalian tissues. Nat. Med. 7, 493-6 (2001)

28. Sköld, K., Svensson, M., Nilsson, A., Zhang, X.Q., Nydahl, K., Caprioli, R.M., Svenningsson, P., Andren, P.E.: Decreased striatal levels of PEP-19 following MPTP lesion in the mouse. J. Proteome Res. 5, 262-269 (2006)

29. Hanrieder, J., Ljungdahl, A., Falth, M., Mammo, S.E., Bergquist, J., Andersson, M.: L-DOPA-induced dyskinesia is associated with regional increase of striatal dynorphin peptides as elucidated by imaging mass spectrometry. Mol. Cell. Proteom 10 . doi:10.1074/mcp.M111.009308 (2011)

30. Monroe, E.B., Annangudi, S.R., Hatcher, N.G., Gutstein, H.B., Rubakhin, S.S., Sweedler, J.V.: SIMS and MALDI MS imaging of the spinal cord. Proteomics 8, 3746-3754 (2008)

31. Cornett, D.S., Frappier, S.L., Caprioli, R.M.: MALDI-FTICR imaging mass spectrometry of drugs and metabolites in tissue. Anal. Chem. 80, 5648-5653 (2008)

32. McDonnell, L.A., Heeren, R.M.A.: Imaging mass spectrometry. Mass Spectrom. Rev. 26, 606-643 (2007)

33. Ye, H., Greer, T., Li, L.: Probing neuropeptide signaling at the organ and cellular domains via imaging mass spectrometry. J. Proteom $\mathbf{7 5}$ 5014-5026 (2012)

34. Rompp, A., Guenther, S., Schober, Y., Schulz, O., Takats, Z., Kummer, W., Spengler, B.: Histology by mass spectrometry: label-free tissue characterization obtained from high-accuracy bioanalytical imaging. Angew. Chem. Int. Ed. 49, 3834-3838 (2010)

35. Klerk, L.A., Altelaar, A.F.M., Froesch, M., McDonnell, L.A., Heeren, R.M.A.: Fast and automated large-area imaging MALDI mass spectrometry in microprobe and microscope mode. Int. J. Mass Spectrom. 285, 19-25 (2009)

36. Jurchen, J.C., Rubakhin, S.S., Sweedler, J.V.: MALDI-MS imaging of features smaller than the size of the laser beam. J. Am. Soc. Mass Spectrom. 16, 1654-1659 (2005)

37. Altelaar, A.F.M., Taban, I.M., McDonnell, L.A., Verhaert, P.D.E.M., de Lange, R.P.J., Adan, R.A.H., Mooi, W.J., Heeren, R.M.A., Piersma,
S.R.: High-resolution MALDI imaging mass spectrometry allows localization of peptide distributions at cellular length scales in pituitary tissue sections. Int. J. Mass Spectrom. 260, 203-211 (2007)

38. Taban, I.M., Altelaar, A.F.M., Van der Burgt, Y.E.M., McDonnell, L.A., Heeren, R.M.A., Fuchser, J., Baykut, G.: Imaging of peptides in the rat brain using MALDI-FTICR mass spectrometry. J. Am. Soc. Mass Spectrom. 18, 145-151 (2007)

39. DeKeyser, S.S., Kutz-Naber, K.K., Schmidt, J.J., Barrett-Wilt, G.A., Li, L.J.: Imaging mass spectrometry of neuropeptides in decapod crustacean neuronal tissues. J. Proteome Res. 6, 1782-1791 (2007)

40. Chen, R.B., Hui, L.M., Sturm, R.M., Li, L.J.: Three Dimensional mapping of neuropeptides and lipids in crustacean brain by mass spectral imaging. J. Am. Soc. Mass Spectrom. 20, 1068-1077 (2009)

41. Verhaert, P.D., Conaway, M.C.P., Pekar, T.M., Miller, K.: Neuropeptide imaging on an LTQ with vMALDI source: the complete 'all-in-one' peptidome analysis. Int. J. Mass Spectrom. 260, 177-184 (2007)

42. Verhaert, P. D., Pinkse, M. W., Strupat, K., Conaway, M. C.: Imaging of similar mass neuropeptides in neuronal tissue by enhanced resolution MALDI MS with an ion trap-Orbitrap hybrid instrument. Methods Mol. Biol. 656, 433-449 (2010)

43. Selverston, A.I., Russell, D.F., Miller, J.P.: The stomatogastric nervous system: structure and function of a small neural network. Prog. Neurobiol. 7, 215-290 (1976)

44. Cape, S.S., Rehm, K.J., Ma, M., Marder, E., Li, L.J.: Mass spectral comparison of the neuropeptide complement of the stomatogastric ganglion and brain in the adult and embryonic lobster, Homarus americanus. J. Neurochem. 105, 690-702 (2008)

45. Li, L.J., Sweedler, J.V.: Peptides in the brain: mass spectrometry-based measurement approaches and challenges. Annu. Rev. Anal. Chem. 1, 451-483 (2008)

46. Kendrick, K.M.: Microdialysis measurement of in vivo neuropeptide release. J. Neurosci. Methods 34, 35-46 (1990)

47. Bungart, D., Dircksen, H., Keller, R.: Quantitative determination and distribution of the myotropic neuropeptide orcokinin in the nervous system of astacidean crustaceans. Peptides 15, 393-400 (1994)

48. Hui, L.M., Zhang, Y.Z., Wang, J.H., Cook, A., Ye, H., Nusbaum, M.P., Li, L.J.: Discovery and functional study of a novel crustacean tachykinin neuropeptide. ACS Chem. Neurosci. 2, 711-722 (2011)

49. Christie, A.E., Cashman, C.R., Brennan, H.R., Ma, M.M., Sousa, G.L., Li, L.J., Stemmler, E.A., Dickinson, P.S.: Identification of putative crustacean neuropeptides using in silico analyses of publicly accessible expressed sequence tags. Gen. Comp. Endocrinol. 156, 246-264 (2008)

50. Nassel, D.R., Winther, A.M.E.: Drosophila neuropeptides in regulation of physiology and behavior. Prog. Neurobiol. 92, 42-104 (2010)

51. Sithigorngul, P., Pupuem, J., Krungkasem, C., Longyant, S., Chaivisuthangkura, P., Sithigorngul, W., Petsom, A.: Seven novel FMRFamide-like neuropeptide sequences from the eyestalk of the giant tiger prawn Penaeus monodon. Comp. Biochem. Phys. B 131, 325-337 (2002)

52. Huybrechts, J., Nusbaum, M.P., Vanden Bosch, L., Baggerman, G., De Loof, A., Schoofs, L.: Neuropeptidomic analysis of the brain and thoracic ganglion from the Jonah crab, Cancer borealis. Biochem. Bioph. Res. Co. 308, 535-544 (2003)

53. Dickinson, P.S., Stemmler, E.A., Cashman, C.R., Brennan, H.R., Dennison, B., Huber, K.E., Peguero, B., Rabacal, W., Goiney, C.C., Smith, C.M., Towle, D.W., Christie, A.E.: SIFamide peptides in clawed lobsters and freshwater crayfish (Crustacea, Decapoda, Astacidea): A combined molecular, mass spectrometric, and electrophysiological investigation. Gen. Comp. Endocrinol. 156, 347-360 (2008)

54. Pipa, R.L.: Studies on the hexapod nervous system. III. Histology and histochemistry of cockroach neuroglia. J. Comp. Neurol. 116, 15-26 (1961)

55. Marshall, A.G., Hendrickson, C.L., Jackson, G.S.: Fourier transform ion cyclotron resonance mass spectrometry: a primer. Mass. Spectrom. Rev. 17, 1-35 (1998)

56. Senko, M.W., Hendrickson, C.L., Emmett, M.R., Shi, S.D.H., Marshall, A.G.: External accumulation of ions for enhanced electrospray ionization Fourier transform ion cyclotron resonance mass spectrometry. J. Am. Soc. Mass Spectrom. 8, 970-976 (1997)

57. Qi, Y.L., Barrow, M.P., Van Orden, S.L., Thompson, C.J., Li, H.L., Perez-Hurtado, P., O'Connor, P.B.: Variation of the Fourier transform mass spectra phase function with experimental parameters. Anal. Chem. 83, 8477-8483 (2011)

58. Chen, R.B., Jiang, X.Y., Conaway, M.C.P., Mohtashemi, I., Hui, L.M., Viner, R., Li, L.J.: Mass spectral analysis of neuropeptide expression 
and distribution in the nervous system of the lobster Homarus americanus. J. Proteome Res. 9, 818-832 (2010)

59. O'Connor, P.B., Costello, C.E.: A high pressure matrix-assisted laser desorption/ionization Fourier transform mass spectrometry ion source for thermal stabilization of labile biomolecules. Rapid Commun. Mass Spectrom. 15, 1862-1868 (2001)

60. da Silva, D., Wasselin, T., Carre, V., Chaimbault, P., Bezdetnaya, L., Maunit, B., Muller, J.F.: Evaluation of combined matrixassisted laser desorption/ionization time-of-flight and matrix- assisted laser desorption/ionization Fourier transform ion cyclotron resonance mass spectrometry experiments for peptide mass fingerprinting analysis. Rapid Commun. Mass Spectrom. 25, 1881-1892 (2011)

61. Römpp, A., Dekker, L., Taban, I., Jenster, G., Boogerd, W., Bonfrer, H., Spengler, B., Heeren, R., Smitt, P.S., Luider, T.M.: Identification of leptomeningeal metastasis-related proteins in cerebrospinal fluid of patients with breast cancer by a combination of MALDI-TOF, MALDIFTICR and nanoLC-FTICR MS. Proteomics 7, 474-481 (2007) 